\section{Sub-relato da ingestão energética e fatores associados em estudo de base populacional}

\author{
Underreporting of energy intake and associated \\ factors in a population-based study
}

\section{Subregistro de la ingesta energética y factores asociados en un estudio de base poblacional}

\author{
Gabriela Ferreira Avelino 1 \\ Ágatha Nogueira Previdelli 1 \\ Michelle Alessandra de Castro 1 \\ Dirce Maria Lobo Marchioni 1 \\ Regina Mara Fisberg ${ }^{1}$
}

\section{Resumo}

This study aimed to identify the prevalence of underreporting of energy intake and associated factors in a sample of 331 individuals from the population-based Health Survey in the City of São Paulo, Brazil. Energy intake was assessed by averaging two 24-hour recalls collected on nonconsecutive days. Total energy expenditure was calculated by the predictive equation, and under-reporters were defined as individuals with energy intake less than 2 standard deviations from the energy intake/predicted total energy expenditure ratio. Poisson regression was used to identify factors associated with underreporting. Prevalence of underreporting of energy intake was $15.1 \%$, whereas individuals with overweight and dissatisfied with body weight were more likely to underreport when compared to individuals without excess weight and who were satisfied with body weight, respectively. We conclude that these factors should be considered when developing techniques to improve the accuracy of dietary assessment.

Energy Intake; Body Weight; Nutrition Surveys
Objetivou-se identificar a prevalência de subrelato da ingestão energética e os fatores associados em amostra de 331 indivíduos, proveniente do estudo de base populacional Inquérito de Saúde no Município de São Paulo, Brasil. A ingestão energética foi avaliada pela média de dois recordatórios de 24 horas coletados em dias não consecutivos. O gasto energético total foi calculado por equação preditiva, considerando sub-relatores os indivíduos com ingestão energética inferior a 2 desvios-padrão da razão ingestão energética/gasto energético total predito. A análise de regressão de Poisson foi utilizada para identificar os fatores associados à subnotificação. A prevalência de sub-relatores da ingestão energética foi de 15,1\%, sendo que os indivíduos com excesso de peso e insatisfeitos com o peso corporal apresentaram maior probabilidade de serem sub-relatores quando comparados aos sem excesso de peso e aos satisfeitos com peso corporal, respectivamente. Conclui-se que esses fatores deveriam ser considerados na elaboração de técnicas para melhorar a acurácia do inquérito dietético.

Ingestão de Energia; Peso Corporal; Inquéritos Nutricionais 


\section{Introdução}

Os dados sobre o consumo alimentar são utilizados como base para recomendações nutricionais, políticas de saúde pública e pesquisas epidemiológicas que estudam as relações entre alimentação e saúde 1 . Porém, um dos mais desafiadores aspectos da ciência e prática da nutrição e dietética é a mensuração do consumo de alimentos, devido às limitações dos métodos para medir essa ingestão acuradamente ${ }^{2}$.

Nesse sentido, um dos principais erros da avaliação dietética está no relato impreciso da ingestão energética, que distorce a interpretação dos resultados de estudos sobre consumo alimentar ${ }^{3}$, sendo mais comum o relato da ingestão energética inferior às quantidades mínimas necessárias para a manutenção do peso corporal do indivíduo, fato conhecido pela literatura científica como sub-relato da ingestão energética. Esse pode ocorrer por lapsos de memória, dificuldade do entrevistado em quantificar as porções, incompreensão das questões feitas pelo entrevistador e até mesmo por constrangimento ao relatar o consumo de alguns alimentos 4,5,6.

Várias características têm sido associadas ao sub-relato em diversos trabalhos, dentre as quais se destacam: sexo, idade, condições socioeconômicas e índice de massa corporal (IMC) 6,7,8,9,10.

No Brasil, o sub-relato da ingestão energética ainda é pouco pesquisado em nível epidemiológico. Sendo assim, este trabalho tem como objetivo identificar a prevalência e os fatores associados ao sub-relato em amostra proveniente de um estudo de base populacional.

\section{Material e métodos}

Foram utilizados os dados do Inquérito de Saúde no Município de São Paulo (ISA-Capital/2008): estudo transversal de base populacional com amostra probabilística de residentes no Município de São Paulo, Brasil 11. Para o presente trabalho foram selecionados indivíduos com 20 anos ou mais, de ambos os sexos, com peso e altura aferidos, e dados completos de dois recordatórios de 24 horas (R24h). Posteriormente, foram excluídos das análises 2 gestantes e 32 sujeitos que declararam mudança recente nos hábitos alimentares, assim, a amostra final foi composta por 331 indivíduos.

A ingestão energética foi obtida pela média dos R24h, coletados em dias não consecutivos e de maneira padronizada por entrevistadores previamente treinados, utilizando-se o MultiplePass Method 12. Esse método consiste em: listagem rápida dos alimentos e horário das refeições, nomeação das refeições, revisão da listagem rápida, ciclo de detalhamento e revisão geral. Os dados obtidos dos R24h foram convertidos em energia e nutrientes pelo software Nutrition Data System for Research (Universidade de Minnesota, Minneapolis, Estados Unidos), que utiliza dados de composição de alimentos da tabela do Departamento de Agricultura dos Estados Unidos, literatura científica e indústria de alimentos.

Para a detecção dos indivíduos sub-relatores utilizou-se a abordagem de McCrory et al. 13, a qual identifica o relato impreciso da ingestão energética por meio da razão Ingestão Energética relatada (IEr)/Gasto Energético Total predito (GETp) pela equação de Vinken et al. 14 e, como pontos de corte, são utilizados \pm 1 desvio-padrão (1DP) ou \pm 2 DP (Figura 1). Neste estudo, foram definidos como sub-relatores os indivíduos com IEr inferior a 2DP da razão IEr/GETp, pois, embora os autores argumentem que o ponto de corte de 1DP seja mais eficaz para excluir sub-relatores da análise, deve-se considerar que a ingestão energética medida representa a ingestão no período de medição, mas não o hábito alimentar. Assim, um ponto de corte muito rigoroso pode superestimar o número de sub-relatores.

As variáveis exploratórias obtidas por questionários estruturados foram: sexo, faixa etária (adultos: 20-59 anos; idosos: $\geq 60$ anos), escolaridade (até 8 anos; $>8$ anos de estudos), renda familiar per capita mensal (até 2; > 2 salários mínimos) e satisfação com o peso corporal (sim; não). O IMC, calculado com base nos dados de peso e altura aferidos, foi utilizado para classificar os indivíduos em: com excesso de peso (IMC $\geq 25$ para adultos e IMC $\geq 27$ para idosos) ou sem excesso de peso (IMC $<25$ para adultos e IMC $<$ 27 para idosos), baseando-se nos critérios propostos pela Organização Mundial da Saúde 15.

Utilizou-se a regressão de Poisson múltipla com variância robusta para verificar os fatores associados ao sub-relato. As variáveis independentes com valor de $\mathrm{p} \leq 0,20$ nas análises bivariáveis foram selecionadas para análise múltipla e incluídas no modelo de regressão por meio do procedimento stepwise forward.

As análises estatísticas foram realizadas no software Stata, versão 11.0 (Stata Corp., College Station, Estados Unidos). Foi considerado nível de significância estatística de $5 \%$.

O presente estudo foi aprovado pelo Comitê de Ética em Pesquisa da Faculdade de Saúde Pública da Universidade de São Paulo (COEP). Todos os participantes assinaram o Termo de Consentimento Livre e Esclarecido. 


\section{Resultados}

Observou-se que $54,1 \%$ de indivíduos apresentaram excesso de peso, destes, 76,5\% relataram insatisfação com o peso corporal. A prevalência de sub-relatores da ingestão energética foi de $15,1 \%$, sendo que aqueles com excesso de peso e insatisfeitos com o peso corporal apresentaram maior probabilidade de serem sub-relatores quando comparados aos indivíduos sem excesso de peso e aos satisfeitos com o peso corporal, respectivamente $(\mathrm{p}<0,05)$ (Tabela 1$)$.

\section{Discussão}

Observou-se prevalência de sub-relato da ingestão energética semelhante à encontrada na literatura internacional e associada ao excesso de peso e insatisfação com o peso corporal. Sendo assim, é importante considerar as características associadas ao sub-relato para melhorar a qualidade dos dados de consumo alimentar.

A prevalência de sub-relato encontrada $(15,1 \%)$ foi semelhante à relatada por McCrory et al. ${ }^{13}$, que também utilizaram o ponto de corte de 2DP e identificaram $11 \%$ de sub-relatores.

Tooze et al. 7, em estudo com idosos, utilizando R24h, também encontraram maior prevalência de sub-relato entre indivíduos com excesso de peso, sendo de $59 \%$ contra $2,6 \%$ entre eutróficos. Parte do baixo consumo energético entre os obesos poderia ocorrer pelo fato de alguns destes indivíduos estarem realizando dietas restritivas no momento do estudo. Entretanto, no trabalho de Bellisle 16, a correlação entre obesidade e subrelato permaneceu mesmo após o ajuste pela prática de dietas, indicando a subnotificação nesse segmento populacional.

Têm sido descritos como contribuintes para o aumento da prevalência de sub-relato em pessoas obesas fatores como insatisfação com a imagem corporal e desejo de ajuste social, que representam a tendência do indivíduo em fornecer uma resposta mais aceitável 17 . Isso reforça os achados do presente estudo, em que os indivíduos insatisfeitos com o peso corporal apresentaram probabilidade $49 \%$ maior de sub-relatar do que os satisfeitos.

Assim, reduzir a prevalência de sub-relato da ingestão energética representa um desafio na área de consumo alimentar, tornando-se necessário o desenvolvimento de estudos sobre técnicas para melhorar a acurácia do inquérito dietético. Uma das alternativas seria um programa de treinamento motivacional como o utilizado por Scagliusi et al. 18. Após o desenvolvimento desse programa, os autores encontraram uma diferença significativa na média do relato de energia entre indivíduos obesos, passando de 1,914kcal para 2,467kcal.

O presente trabalho teve como limitação o uso de equações preditivas para detectar o sub-relato, contudo, a utilização de métodos como a água duplamente marcada é dificultada em estudos de base populacional devido ao seu elevado custo e complexidade.

Assim, existe a necessidade de desenvolvimento de métodos viáveis de serem aplicados em estudos de base populacional. Isso se torna mais relevante quando a população tem elevada prevalência de excesso de peso, pois o mesmo associase tanto à maior ocorrência de doenças crônicas não transmissíveis quanto à maior probabilidade de sub-relato.

Tabela 1

Distribuição da amostra, prevalência e razão de prevalência (RP) de sub-relatores da ingestão energética segundo características da população de estudo. São Paulo, Brasil, 2008.

\begin{tabular}{|c|c|c|c|c|c|c|}
\hline \multirow[t]{2}{*}{ Variáveis } & \multirow{2}{*}{$\begin{array}{c}\text { População } \\
\text { total } \\
\text { n (\%) }\end{array}$} & \multirow{2}{*}{$\begin{array}{c}\text { Indivíduos } \\
\text { sub-relatores } \\
\text { n (\%) }\end{array}$} & \multicolumn{2}{|c|}{ Regressão simples } & \multicolumn{2}{|c|}{ Regressão múltipla } \\
\hline & & & RP (IC95\%) & Valor de $\mathrm{p}$ & RP (IC95\%) & Valor de $p$ \\
\hline \multicolumn{7}{|l|}{ Estado nutricional } \\
\hline Sem excesso de peso & $152(45,92)$ & $14(28,00)$ & 1,00 & & 1,00 & \\
\hline Com excesso de peso & $179(54,08)$ & $36(72,00)$ & $1,80(1,00-3,27)$ & 0,052 & $2,33(1,23-4,41)$ & 0,010 \\
\hline \multicolumn{7}{|c|}{ Satisfação com o peso corporal } \\
\hline Não & $137(41,64)$ & $25(50,00)$ & 1,00 & & 1,00 & \\
\hline Sim & $192(58,36)$ & $25(50,00)$ & $0,71(0,41-1,24)$ & 0,200 & $0,51(0,28-0,94)$ & 0,030 \\
\hline
\end{tabular}

(continua) 
Tabela 1 (continuação)

\begin{tabular}{|c|c|c|c|c|c|c|}
\hline \multirow[t]{2}{*}{ Variáveis } & \multirow{2}{*}{$\begin{array}{l}\text { População } \\
\text { total } \\
\text { n (\%) }\end{array}$} & \multirow{2}{*}{$\begin{array}{c}\text { Indivíduos } \\
\text { sub-relatores } \\
\text { n (\%) }\end{array}$} & \multicolumn{2}{|c|}{ Regressão simples } & \multicolumn{2}{|c|}{ Regressão múltipla } \\
\hline & & & RP (IC95\%) & Valor de $p$ & RP (IC95\%) & Valor de $p$ \\
\hline \multicolumn{7}{|l|}{ Sexo } \\
\hline Masculino & $129(38,97)$ & $24(48,00)$ & 1,00 & & & \\
\hline Feminino & $202(61,03)$ & $26(52,00)$ & $0,69(0,40-1,20)$ & 0,209 & - & - \\
\hline \multicolumn{7}{|c|}{ Escolaridade (anos completos de estudo) } \\
\hline Até 8 & $128(38,91)$ & $34(69,39)$ & 1,00 & & & \\
\hline$>8$ & $201(61,09)$ & $15(30,61)$ & $0,87(0,41-1,26)$ & 0,251 & - & - \\
\hline \multicolumn{7}{|l|}{ Faixa etária } \\
\hline Adultos & $173(52,27)$ & $28(56,00)$ & 1,00 & & & \\
\hline Idosos & $158(47,73)$ & $22(44,00)$ & $0,86(0,49-1,50)$ & 0,597 & - & - \\
\hline \multicolumn{7}{|c|}{ Renda familiar per capita * (salários mínimos) } \\
\hline Até 2 & $235(74,13)$ & $36(76,60)$ & 1,00 & & & \\
\hline 2 ou mais & $82(25,87)$ & $11(23,40)$ & $0,87(0,44-1,72)$ & 0,700 & - & - \\
\hline
\end{tabular}

* Considerou-se o valor do salário mínimo de $\mathrm{R} \$ 415,00$, vigente no ano de 2008.

Figura 1

Abordagem de McCrory et al. 13 para detecção de indivíduos sub-relatores da ingestão energética.

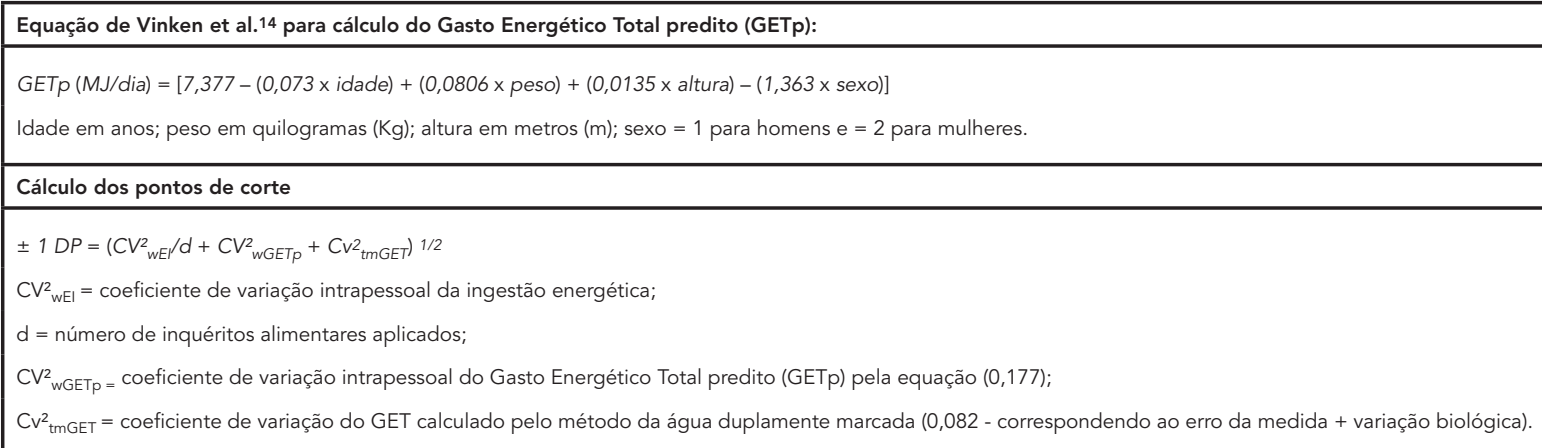




\section{Resumen}

El presente estudio tuvo por objetivo identificar la prevalencia del subregistro de ingesta energética y sus factores asociados, en una muestra de 331 individuos pertenecientes al estudio de base poblacional Encuesta de Salud en el Municipio de São Paulo, Brasil. La evaluación fue realizada a través de dos recordatorios de 24 horas en días no consecutivos. El gasto energético total por individuo se calculó mediante ecuación predictiva y fue considerado subregistro, cuando la ingesta energética fuera inferior a 2 desviaciones estándar de la razón ingesta energética/gasto energético total. Con el fin de identificar los factores asociados al subregistro, se utilizó el análisis de regresión múltiple. La prevalencia de subrelato fue de un 15,1\%, teniendo en cuenta que individuos con exceso de peso e insatisfechos con el peso corporal presentaron mayor probabilidad de ser subrelatores, comparados con los individuos sin exceso de peso y los satisfechos con su peso corporal, respectivamente. Se concluye que estos factores deberían considerarse en la elaboración de técnicas para mejorar la precisión de las encuestas dietéticas

Ingestión de Energía; Peso Corporal; Encuestas

Nutricionais

\section{Colaboradores}

G. F. Avelino participou da análise e interpretação de dados e redação do manuscrito. Á. N. Previdelli e M. A. Castro participaram da análise de dados e revisão crítica do manuscrito. D. M. L. Marchioni e R. M. Fisberg participaram da revisão crítica do manuscrito.

\section{Agradecimentos}

À Fapesp (processo no 2009/15831-0), ao CNPq (processo no 473100/2009-6) e à Secretaria Municipal de Saúde de São Paulo pelo financiamento do estudo.

\section{Referências}

1. Hill RJ, Davies PSW. The validity of self-reported energy intake as determined using doubly labeled water technique. Br J Nutr 2001; 85:415-30.

2. Fisberg RM, Colucci ACA, Morimoto JM, Marchioni DML. Questionário de freqüência alimentar para adultos com base em estudo populacional. Rev Saúde Pública 2008; 42:550-4.

3. Black AE, Bingham SA, Johansson G, Coward WA. Validation of dietary intakes of protein and energy against 24 hour urinary N and DLW energy expenditure in middle-aged women, retired men and post-obese subjects: comparisons with validation against presumed energy requirements. Eur J Clin Nutr 1997; 51:405-13.

4. Margetts BM, Nelson M. Design concepts in nutritional epidemiology. New York: Oxford University Press; 1991.

5. Tomoyasu NJ, Toth MJ, Poehlman ET. Misreporting of total energy intake in older men and women. J Am Geriatr Soc 1999; 47:710-5.
6. Krestch MJK, Fong AKH, Green MW. Behavioral and body sizes correlates of energy intake underreporting by obese and normal-weight women. J Am Diet Assoc 1999; 99:300-6.

7. Tooze JA, Vitolins MZ, Smith SL, Arcury TA, Davis CC, Bell RA, et al. High levels of low energy reporting on 24-hour recalls and three questionnaires in an elderly low-socioeconomic status population. J Nutr 2007; 137:1286-93.

8. Scagliusi FB, Ferriolli E, Pfrimer K, Laureano C, Cunha CS, Gualano B, et al. Underreporting of energy intake in brazilian women varies according to dietary assessment: a cross-sectional study using doubly labeled water. J Am Diet Assoc 2008; 108:2031-40.

9. Scagliusi FB, Ferriolli E, Pfrimer K, Laureano C, Cunha CS, Gualano B, et al. Characteristics of women who frequently under report their energy intake: a doubly labelled water study. Eur J Clin Nutr 2009; 63:1192-9. 
10. Ferriolli E, Pfrimer K, Moriguti JC, Lima KCN, Moriguti EKU, Formighieri PF, et al. Under-reporting of food intake is frequent among Brazilian freeliving older persons: a doubly labelled water study. Rapid Commun Mass Spectrom 2010; 24:506-10.

11. Cesar CLG, Segri NJ, Sportello RA. Inquéritos de saúde no Estado de São Paulo. São Paulo: Faculdade de Saúde Pública, Universidade de São Paulo; 2005.

12. Conway JM, Ingwersen LA, Vinyard BT, Moshfegh AJ. Effectiveness of the US Department of Agriculture 5-step multiple-pass method in assessing food intake in obese and nonobese women. Am J Clin Nutr 2003; 77:1171-8.

13. McCrory MA, Hajduk C, Roberts SB. Procedures for screening out inaccurate reports of dietary energy intake. Public Health Nutr 2002; 5:873-82.

14. Vinken AG, Bathalon GP, Sawaya AL, Dallal GE, Tucker KL, Roberts SB. Equations for predicting the energy requirements of healthy adults aged 1881y. Am J Clin Nutr 1999; 69:920-6.
15. World Health Organization. Obesity: preventing and managing the global epidemic: report of a consultation. Geneva: World Health Organization; 2000. (WHO Technical Report Series).

16. Bellisle F. The doubly-labeled water method and food intake surveys: a confrontation. Rev Nutr 2001; 14:125-33.

17. Hebert JR, Clemow L, Pbert L, Ockene IS, Ockene JK. Social desirability bias in dietary self-report may compromise the validity of Dietary Intake Measure. Int J Epidemiol 1995; 24:389-98.

18. Scagliusi FB, Polacow VO, Artioli GG, Benatti FB, Lancha Jr. AH. Selective underreporting of energy intake in women: magnitude, determinants and effect of training. J Am Diet Assoc 2003; 103:1306-13.

Recebido em 26/Mar/2013

Versão final reapresentada em 22/Out/2013

Aprovado em 04/Nov/2013 